\title{
Un atlas linguistique du créole des Petites Antilles
} (ALPA)

A linguistic atlas of Lesser Antillean French Creole

Jean Le Dû

\section{OpenEdition}

\section{Journals}

Édition électronique

URL : https://journals.openedition.org//bl/1551

DOI : $10.4000 / \mathrm{lbl} .1551$

ISSN : 2727-9383

\section{Éditeur}

Université de Bretagne Occidentale - UBO

\section{Édition imprimée}

Date de publication : 1 novembre 2011

Pagination : 59-74

ISBN : 978-2-901737-92-6

ISSN : $1270-2412$

\section{Référence électronique}

Jean Le Dû, « Un atlas linguistique du créole des Petites Antilles (ALPA) », La Bretagne Linguistique [En ligne], 16 | 2011, mis en ligne le 01 mai 2021, consulté le 22 mai 2021. URL : http://

journals.openedition.org//bl/1551; DOI : https://doi.org/10.4000/lbl.1551

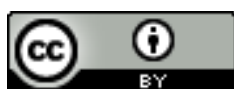

La Bretagne Linguistique est mise à disposition selon les termes de la Licence Creative Commons Attribution 4.0 International. 
Jean LE DÛ

\title{
Un atlas linguistique du créole des Petites Antilles (ALPA)
}

\begin{abstract}
$\coprod^{9}$
ai assuré durant les années 1990 à la Faculté des Lettres de l'Université des Antilles et de la Guyane (campus de Schœlcher, Martinique), à l'invitation de Jean Bernabé, une introduction à la dialectologie et à la géolinguistique dans le cadre de la licence de créole. Comme il n'y avait pas de géolinguiste dans l'équipe, Jean Bernabé m'a proposé en 1998 de prendre en charge la réalisation d'un atlas de l'espace créolophone antillais. J'ai alors rédigé un questionnaire que j'ai revu avec l'aide du créoliste Robert Damoiseau, qui a organisé la campagne d'enquêtes. En 1999, j'ai formé des étudiants volontaires au travail de terrain et à la notation des données en phonétique. J'avais envisagé, au départ, d'inclure la Guyane dans l'atlas, mais il s'est avéré que nous n'avions pas les moyens d'y réaliser des enquêtes. Étant donné l'étendue de la zone et les énormes difficultés de déplacement, il semble préférable d'organiser un chantier propre à cette région. La zone retenue constitue une unité géographique, historique et humaine remarquable. Une enquête de contrôle a cependant été réalisée sur le continent sud-américain par Jo-Anne Ferreira, une collègue linguiste de l'Université des Indes Occidentales (Uni-
\end{abstract}

* Professeur émérite de celtique, CRBC, UBO/ueb 
versity of the West-Indies). Elle y a interrogé des Indiens karipúna et Garibi-Marworno qui ont comme langue maternelle le créole (kheuol, patoa) vivant dans la région d'Oiapoque, une petite ville limitrophe située dans l'État d'Amapá, au Brésil. Elle n'a malheureusement pas eu le temps de transcrire les données d'une autre enquête réalisée dans la presqu'île de Paria, au Venezuela, où le créole, jadis introduit de Trinité, est en voie d'abandon.

\section{Présentation du terrain d'enquête}

Les Petites Antilles (par opposition aux Grandes Antilles : Cuba, la Jamaïque, Hispaniola - Haïti et Saint-Domingue -, Porto Rico), orientées sur un axe nord-sud, reçoivent régulièrement les vents alizés du nord-est, d'où leur nom «Îles du vent» (les «Îles au vent», principalement néerlandaises, suivent une orientation parallèle à la côte du continent).

Les «côtes au vent», du côté atlantique, sont constamment agitées, tandis que les côtes caraïbes, dites «sous le vent», sont abritées ${ }^{1}$. Le climat tropical connaît deux saisons : la période cyclonique ou «hivernage», de juin à décembre, humide et chaude, et la période du «carême», de décembre à juin, sèche et plus fraîche.

Politiquement, cinq pays sont concernés : la France, trois pays du Commonwealth - la Dominique, Sainte-Lucie et Trinité-et-Tobago - et le Brésil. Faute de moyens, il n'a pas été possible d'enquêter à Saint-Vincent-et-Grenadines ni à la Grenade, où subsisteraient des traces de créole français ${ }^{2}$. D'autres créoles français d'Amérique se parlent aussi en Louisiane, en Haïti et dans quelques poches disséminées dans divers pays, par exemple au Panamá ${ }^{3}$.

1. Les Anglais considèrent que Saint-Martin, Saint-Barthélemy et la Guadeloupe font partie des îles sous le vent (Leeward Islands), parce qu'elles ne sont pas directement exposées aux alizés (trade winds).

2. Je n'ai jamais reçu de réponse à mes demandes de renseignements adressés à divers organismes de ces pays : il est probable que l'état du créole ne fait pas partie de leurs préoccupations premières.

3. Le 16 août 2005 un avion provenant du Panamá avec à bord 152 passagers martiniquais s'est écrasé au Venezuela. Ces gens venaient de rendre visite à la diaspora martiniquaise descendant de personnes qui avaient émigré au XIX ${ }^{\mathrm{e}}$ siècle pour participer à la construction du Canal. La plupart ne parlent plus le créole, mais ont conservé des traditions de leur pays d'origine. 


\section{Le peuplement des Isles}

\section{Les Français}

Les Français, arrivés au XVII ${ }^{\mathrm{e}}$ siècle, ont trouvé sur place les Caraïbes $^{4}$, une population autochtone qui a disparu sous l'effet des maladies importées et surtout en raison du travail forcé 5 . Ils ont laissé des mots en créole et en français (ananas, hamac, tabac, etc.).

Ceux des colons qui ont payé leur voyage deviennent des «maîtres de case». Ils font venir de métropole de la main-d'œuvre, les «engagés», qui devaient travailler pendant trente-six mois pour eux afin de rembourser leur voyage aller et retour. Ces gens miséreux s'expatriaient en raison des famines ou de la grande pauvreté qui les frappait. Ils étaient majoritairement originaires de l'ouest et du nord-ouest de la France ${ }^{6}$, particulièrement du Poitou, d'Aunis et de Saintonge, mais aussi d'Anjou, Bretagne, Normandie, Île de France, etc. Il s'agissait essentiellement de ruraux, mais on trouvait également parmi eux des artisans (charpentiers, menuisiers, forgerons, etc.). L'origine de ces gens est importante pour comprendre la genèse du créole : ils ne parlaient pas le français que nous connaissons actuellement mais des «patois» variés, parlers locaux qui ont subsisté longtemps et ont été en grande partie étudiés dans l'Atlas Linguistique de la France de Gilliéron et Edmont et dans les atlas linguistiques et ethnographiques régionaux du $\mathrm{XX}^{\mathrm{e}}$ siècle.

\section{L'esclavage}

On trouve des Noirs dès les débuts dans l'économie dite «d'habitation", au temps où la culture du tabac ne demandait pas trop de main-d'œuvre: ils étaient à ce stade en nombre inférieur aux Blancs.

4. Amérindiens originaires du bassin de l'Orénoque arrivés vers le $\mathrm{X}^{\mathrm{e}}$ siècle.

5. Il en reste une réserve à la Dominique, de langue créole, métissée de noirs. Mythe de la lutte contre les Arawaks, dont ils auraient massacré et mangé les hommes (caniba $=$ cannibale) et épousé les femmes, qui parlaient une langue différente. Justification de la colonisation.

6. Gabriel Debien, Les engagés pour les Antilles (1634-1715), Société de l'histoire des colonies françaises, Paris, 1952. 
L'économie dite de «plantation» étant très gourmande en maind'œuvre, des esclaves sont importés d'Afrique pour la faire fructifier. À partir de 1680, les armateurs de bateaux trouveront cette activité plus rentable... Le «commerce triangulaire» devient au XVII siècle une source immense de profit pour les armateurs et épargnants européens ainsi que pour leurs fournisseurs africains : des navires partent de Bordeaux, de Nantes, etc. chargés de verroterie, d'alcool, d'armes qu'ils échangent dans les comptoirs d'Afrique contre des esclaves fournis par des chefs locaux ${ }^{7}$. (À Abomey, au Bénin, on peut voir exposés au musée des canons dont le prix est évalué au nombre d'esclaves qu'ils ont coûté.) Ces navires vont alors en Amérique échanger leur cargaison humaine contre des produits locaux (sucre, tabac, coton, métaux précieux) qu'ils retournent vendre en Europe. Tout cela sera géré par le Code noir promulgué en 1685 par Louis XIV : on y précise que Les esclaves sont des biens meubles, ce qui n'empêche pas leur conversion forcée au christianisme!

L'esclavage a été aboli le 4 février 1794 grâce à l'action de l'abbé Grégoire, puis restauré par Napoléon en 1802 : Grégoire ${ }^{8}$ a continué son action anti-esclavagiste avec son appel De la traite et de l'esclavage des Noirs et des Blancs. Le deuxième décret d'abolition de l'esclavage a été signé le 27 en 1848 par le poète Lamartine sous l'impulsion de Victor Schœlcher.

\section{Les travailleurs volontaires}

Les esclaves affranchis quittent alors les plantations. Pour les remplacer, on fait venir des travailleurs africains «volontaires» du Congo (les «Nègres Congo»). À partir de 1861, est organisée une immigration indienne (appelés «coolies» en Martinique, «malabars » à la Guadeloupe). À la fin du XIX siècle, des commerçants chinois, libanais, syriens arrivent à leur tour.

Les langues africaines ont été abandonnées par les esclaves, mais sans doute pas aussi rapidement qu'on a pu le penser. Cependant,

7. On comprend le sentiment de rancœur des Antillais à l'égard des Africains...

8. Cet humaniste a également milité pour que les Juifs se voient accordée la citoyenneté française. Il est haï par des défenseurs des langues régionales à cause de son Rapport sur la Nécessité et les Moyens d'anéantir les Patois et d'universaliser l'Usage de la Langue française. 
toutes ces personnes d'origines géographiques différentes avaient besoin d'un parler commun, le créole. Des souvenirs d'Afrique ont subsisté : la musique, la danse, quelques mots et aussi des pratiques religieuses qui ont survécu à la christianisation forcée en donnant naissance au quimbois (nom antillais du vaudou), religion syncrétique. Toute la vie des habitations tournait autour du travail, dans un cadre nouveau, sans vrai contact avec les maîtres blancs ni avec les autres communautés d'esclaves.

\section{Inventaire des îles enquêtées}

Voici une brève description des îles dans lesquelles s'est déroulée l'enquête :

- Saint-Martin (pt 01, 35000 habitants) est située à $230 \mathrm{~km}$ au nord-ouest de la Guadeloupe et à $25 \mathrm{~km}$ au nord de Saint-Barthélemy. Elle est partagée entre la France (partie nord) et les Pays-Bas (partie sud, Sint-Maarten en néerlandais) depuis 1648, et l'anglais y sert de langue véhiculaire. La partie française est une Collectivité d'Outre-mer (COM) depuis 2007.

- Saint-Barthélemy (pt 02, 8000 habitants) est située à $200 \mathrm{~km}$ au nord-ouest de la Guadeloupe et à $25 \mathrm{~km}$ au sud de Saint-Martin. Conquise par la France en 1648, elle fut suédoise de 1784 à 1876, avant de revenir à la France. Surtout connue pour son tourisme de luxe, elle est COM depuis 2007. Dans la partie au vent on parle créole tandis que dans la partie sous le vent subsiste un «patois » archaïque (Calvet et Chaudenson, 1998) qui, note l'enquêtrice Madjanie Leprix, est parlé par la vieille génération, qui est réticente à l'utiliser devant des étrangers. Elle en a relevé quelques phrases : Vèy, lapli ki va tonbé «regarde (veille), il va pleuvoir»; Di mwé sak ta vu «dis-moi ce que tu as vu». L'influence du créole s'y fait sentir : Léswa j'manj pa empil «le soir je ne mange pas beaucoup».

- La Guadeloupe (pts 03-07, 09-15, 451000 habitants) est la réunion de Basse-Terre à l'ouest et Grande-Terre à l'est, reliées par un pont. Découverte par Christophe Colomb en 1493, elle devint française en 1635. Département français d'outre-mer (DOM) depuis 1946, la Guadeloupe a le statut de DROM (Département et Région d'Outre-mer) depuis 2003. Ses dépendances administratives actuelles sont réduites à Marie-Galante, la Désirade et les Saintes. 
- La Désirade (pt 08, 1595 hab.) est une île calcaire, aride de forme allongée (11 km sur $2 \mathrm{~km})$.

- La Dominique (pts 19-26, 72000 habitants), Dominica en anglais, est située entre la Guadeloupe et la Martinique. Découverte par Christophe Colomb en 1493, elle est d'abord peuplée par des Français mais devient anglaise par le traité de Paris (1763). Membre du Commonwealth et des Nations Unies, elle est indépendante depuis 1978. Elle est la seule des îles à conserver une petite population d'origine caraïbe, souvent métissée.

- Marie-Galante (pt 16, 13500 habitants) est située à $25 \mathrm{~km} \mathrm{au}$ sud-est de l'île de Basse-Terre. Découverte par Christophe Colomb en 1493, elle est partagée entre trois communes. Formée d'un plateau calcaire, elle a une forme circulaire.

- Les Saintes (pts 17-18) constituent un archipel dont deux îles sont peuplées : Terre-de-Bas (1 030 hab.) et Terre-de-Haut $\left(5 \mathrm{~km}^{2}\right.$, 1800 habitants). Découvertes par Christophe Colomb en 1493, françaises depuis 1648, elles sont peuplées majoritairement de descendants de Bretons, Charentais et Normands. Le créole y a un aspect particulier dont le trait le plus immédiatement observable est la préservation de voyelles arrondies : [ko] 'cœur' au lieu de [ke].

- La Martinique (pts 27-38, 400000 habitants). Découverte par Christophe Colomb en 1502, elle devient française en 1635 et est réunie à la Couronne en 1674. De 1794 à 1802, l'île est sous occupation anglaise, puis à nouveau de 1809 à 1814, avant de redevenir française en 1815. Érigée en DOM en 1946, l'île devient en 2003 un DROM.

- Sainte-Lucie (pts 39-46, 173000 habitants) s'appelle en anglais Saint Lucia. Elle est située entre la Martinique au nord et Saint-Vincent-et-les-Grenadines au sud. Après l'établissement d'une colonie française en 1635, l'île a fait l'objet de rivalités constantes entre Français et Anglais jusqu'en 1814, quand le traité de Paris l'a rattachée à l'Angleterre. Membre du Commonwealth, Sainte-Lucie est indépendante depuis 1979.

- L'île de la Trinité (Trinidad) (pt 47, environ 1 million d'habitants) fait partie de l'État de Trinidad-and-Tobago, capitale Portof-Spain. Situé au nord de l'embouchure de l'Orénoque, elle est à $11 \mathrm{~km}$ des côtes du Venezuela. Découverte par Christophe Colomb en 1493, elle est devenue une possession espagnole. Au moment 
de la Révolution française, de nombreuses familles françaises s'y sont installées avec leurs esclaves, y introduisant le français et le créole, qui est devenu pour un siècle la véritable lingua franca de l'île - qui n'a pourtant jamais été une possession française. En 1802, l'île devient officiellement anglaise, et s'unit à Tobago pour constituer en 1962 un État indépendant membre du Commonwealth. L'esclavage étant aboli en 1838, on fait venir entre 1845 et 1917 plus de 150000 Indiens pour les remplacer, ce qui fait que les populations d'origine indienne et africaine sont à peu près à égalité. Le créole français, de même que le bhojpuri (langue de l'Inde) sont en voie d'abandon, tandis que l'espagnol serait encore utilisé par quelques pêcheurs. L'anglais et un créole anglais sont parlés de nos jours par la grande majorité de la population. On trouve cependant un nombre non négligeable de locuteurs de créole français, mais la plupart sont assez âgés (un locuteur natif de 26 ans, Marvel Henry, m'a aidé dans l'interprétation de certaines données).

- La péninsule de Paria a été peuplée par des immigrants de la Trinité fuyant les tracasseries du pouvoir anglais. Ils y ont introduit le créole (patuá), jadis florissant mais aujourd'hui en voie de disparition au profit de l'espagnol.

- Oiapoque, ville de l'État de l'Amapá (Brésil) fait face à la ville française de Saint-Georges-de-l'Oyapock en Guyane. Le créole est la langue maternelle de groupes indiens (Karipúna et Galibi-Marwono) et sert aussi de langue véhiculaire (Calvet, 2009).

\section{Présentation linguistique}

\section{Les pidgins}

Il y a souvent dans le grand public et aussi chez certains linguistes confusion entre pidgins et créoles. Les pidgins sont par définition des parlers transitoires servant à la communication entre groupes humains ne partageant aucune langue commune. Ils ne sont pas langues maternelles, bien que certains le soient devenus, par exemple le tok pisin, actuellement langue officielle en PapouasieNouvelle Guinée. Le mot viendrait de business prononcé à la chinoise (contacts entre anglophones et sinophones). On connaît la lingua franca (langue franque) de Méditerranée (ou sabir) utilisée jusqu'au $\mathrm{XIX}^{\mathrm{e}}$ siècle par les populations de toutes origines, le Russenorsk qui a 
servi aux échanges entre pêcheurs norvégiens et commerçants russes aux XVIII et $\mathrm{XIX}^{\mathrm{e}}$ siècles jusqu'à la Révolution d'octobre. On peut considérer que ces façons de parler sont nées entre communautés de statut égal.

\section{Théories sur la naissance du créole}

Les créoles, en revanche, sont apparus dans des conditions de domination. Je ne parlerai que du créole français des Antilles, but de la recherche que je présente ici. Cette langue, issue du français des immigrants, s'est individualisée à partir du XVII et surtout du XVIII ${ }^{\mathrm{e}}$ siècle dans les plantations coloniales (Chaudenson, 2000).

La tendance - toujours dominante - est de considérer les créoles comme des langues à part, différentes des langues "naturelles», d'où la science qui s'appelle la créolistique (creolistics apparaît en anglais vers 1960 et créolistique en français vers 1980). Ces langues constituent pour certains théoriciens du langage un terrain d'expérimentation. Leur localisation dans des territoires exotiques n'est sans doute pas pour rien dans leur succès. Ce point de vue, dit exceptionnaliste, est rejeté par un certain nombre de spécialistes (DeGraff, 2005).

On sait que le problème des origines a toujours joué un rôle important dans le développement de la linguistique, nous sommes bien placés pour le savoir, avec l'éternel questionnement sur l'origine insulaire ou continentale du breton...

Je ne mentionnerai que quelques-unes des nombreuses théories qui donnent lieu à des discussions et à des confrontations incroyablement animées entre spécialistes.

1. La théorie monogénétique: Hugo Schuchardt, considéré comme le créateur des études créoles (et basques), a avancé l'idée que tous les créoles viendraient d'une même source, le pidgin portugais parlé sur les côtes d'Afrique et répandu ensuite dans les diverses colonies par les Portugais, grands organisateurs de la traite des Noirs à travers le monde, avec relexification. D'autres tenants d'une théorie monogénétique ont proposé que la lingua franca de Méditerranée serait à la base des créoles.

2. La théorie substratiste : d'autres chercheurs croient à la forte influence du substrat de langues africaines. Ainsi Claire Lefèvre 
(1999), qui soutient que le haïtien serait une relexification du fongbe, langue du Bénin et du Togo actuels.

3. La théorie innéiste : le linguiste Derek Bickerton considère qu'il existe un «bioprogramme» - une prédisposition génétique à la parole - qui se manifeste au moment où des enfants font du pidgin leur langue maternelle en la simplifiant et en l'adaptant à leur capacité universelle de parler. Ainsi s'expliquerait la ressemblance structurale entre tous les créoles. Ces langues jeunes seraient donc les manifestations proches des débuts de l'humanité. Une objection vient immédiatement à l'esprit: la société dans laquelle le créole a pris naissance était composée de personnes jeunes, mais ne comportait ni enfants, ni vieillards...

4. La théorie gradualiste : pour Robert Chaudenson, la créolisation est une approximation d'approximations de variétés de français populaires du XVII ${ }^{\mathrm{e}}$ siècle. Le langage des sociétés d'habitation, ayant cédé la place aux sociétés de plantation, a subi une série de changements de la part des esclaves : ce sont les plus anciens esclaves, ayant désormais peu de contacts avec les Blancs, qui enseignaient la langue aux nouveaux venus, les bossales. Salikoko Mufwene (2003) pense que les créoles devraient être considérés comme des continuations des langues à partir desquelles ils se sont développés et que leur étude ressortit de la linguistique historique.

5. La théorie du prototype créole : défendue par McWhorter et Parkvall (1998, 2002), elle argue qu'un certain nombre de faits de simplification, individuellement présents dans d'autres langues, ne se trouvent réunis que dans les créoles, ce qui s'expliquerait par un stade préalable de pidgin : absence de flexion, absence de tons lexicaux, dérivation sémantiquement transparente...

\section{Situation sociolinguistique du créole antillais}

Étant donné la situation sociale des locuteurs, on trouve encore des monoglottes, surtout dans les îles ex-anglaises, économiquement moins développées. Dans les îles françaises, la tendance est au rapprochement vers la langue nationale - on parle alors de décréolisation - ce qui s'observe aussi dans les parlers régionaux comme le gallo. Ce phénomène est particulièrement marqué en Martinique. Dans une 
interview publiée sur le site Montray $\mathrm{Kreyol}^{9}$, Madjanie Leprix - qui a réalisé à elle seule la moitié des enquêtes pour l'Atlas Linguistique des Petites Antilles - affirme que :

«Vous comprenez bien qu'avec un père agriculteur né dans les années 30, et lui-même fils d'agriculteur, la langue qui primait à la maison était celle de la société de l'époque, autrement dit, celle de la société d'habitation. Quand je retourne au pays me ressourcer, je deviens un autre Moi. Autrement dit, en Martinique, mis à part au travail et avec quelques rares ami(e)s, je m'exprime essentiellement en langue française. En Guadeloupe, c'est le processus inverse. Je respire et vis pleinement le créole. Je n'échange que très, très rarement en français.»

Plus qu'en Guadeloupe, les parents tendent à exclure l'usage du créole de la maison, ce qui fait que cette langue ne s'acquiert apparemment plus chez les enfants qu'au contact des groupes de pairs. C'est ce qu'a montré Christian March dans sa thèse (March, 1996) sur le discours des mères martiniquaises. On oppose basilecte, la forme de créole la plus éloignée du standard, à l'acrolecte, le plus proche du standard, et au mésolecte, variété intermédiaire.

Sainte-Lucie, comme la Dominique, vit une situation de diglossie créole/ anglais, cette dernière langue prenant une place de plus en plus grande chez les jeunes.

La zone antillaise est un vrai laboratoire du point de vue scientifique : un créole français est parlé dans une série d'îles voisines dont deux ont pour langue officielle le français, trois autres l'anglais (et naguère l'espagnol à Trinité) et enfin le portugais. Comme la diglossie est par nature inséparable de la situation des créoles - l'unilinguisme de beaucoup de Haïtiens étant une affaire de sous-développement - cette exposition à une langue-toit autre que le français, si elle entraîne quelques calques et emprunts, favorise un conservatisme du vieux fonds lexical. Les îles françaises sont, en effet, exposées directement au français par l'école et les médias, ce qui amène à un renouvellement du lexique. La comparaison des données permet donc une datation relative - nous en verrons des exemples plus bas - en séparant les emprunts français récents du fonds lexical le plus

9. https://www.montraykreyol.org/article/madjanie-leprix-sur-amina 
ancien. De façon générale, les locuteurs du créole sont en majorité des ruraux. Depuis les années 1950, les parents des centres urbains, soucieux de voir leurs enfants s'exprimer en anglais, la langue officielle, qui est perçue comme un moyen de mobilité sociale, ont découragé l'utilisation du créole. De plus en plus de Sainte-Luciens ont l'anglais comme langue maternelle. En outre, l'influence de l'anglais est plus marquée aujourd'hui dans le vocabulaire technique et les expressions de la vie quotidienne. Les divergences se renforcent ainsi progressivement entre le créole de Sainte-Lucie et celui de la Martinique, lui-même très marqué par le français moderne.

\section{Quelques notions de base sur la langue}

Je ne dirai que quelques mots sur la langue elle-même, en m'attachant aux faits qui m'ont frappé quand j'ai commencé à l'étudier.

La langue est appelée «patois» par ses locuteurs, même dans les pays de langue anglaise et espagnole ${ }^{10}$. Bien qu'incompréhensible pour un francophone, elle est fortement apparentée au français du point de vue lexical. L'article français et le partitif d'origine sont souvent amalgamés au nom : lapòt 'porte', diri 'riz', dlo 'eau'...

Du point de vue phonétique, il n'y a pas de voyelles arrondies (mi 'mûr', flè 'fleur', blé 'bleu'); les voyelles sont nasales devant les consonnes nasales (flanm 'flamme', menm 'même') comme en breton.

Du point de vue morphologique, on constate que pratiquement tous les éléments sont invariables. Pour le groupe nominal, il existe un article indéfini on ou an; l'article défini est postposé : dlo la 'l'eau'. Le pluriel se forme avec se ... la (se flè la 'les fleurs'), et le démonstratif en ajoutant une particule (se flè tala 'ces fleurs-là').

Le groupe verbal se caractérise par une conjugaison à l'aide de particules (un peu comme le futur de l'anglais shall et will) : mwen $d i$ 'j'ai dit', mwen $k a$ di 'je dis', mwen ké di 'je dirai', mwen té di 'je disais', mwen té ka di 'j'étais en train de dire', mwen té ké di 'je dirais'...

10. L'origine du mot créole est discutée : s'applique-t-il aux seuls Blancs? à tout ce qui est né dans les Isles, y compris les Noirs et même les animaux plantes et objets? Il a été repris par un mouvement de la «créolité» avec Jean Bernabé, Raphaël Confiant, Patrick Chamoiseau, etc. 


\section{Présentation de l'ALPA}

\section{Questionnaire}

Le questionnaire comprend 467 questions organisées en rubriques (météo, nature, espace, vie sociale, etc.). Il comprend des mots isolés (ananas, bicyclette), des courtes phrases (il fait chaud), quelques phrases plus longues (le voleur a sauté par-dessus le mur) et quelques questions ouvertes (phénotypes, sortes de crabes, etc.).

\section{Enquêteurs et transcriptions}

Les enquêtes et les transcriptions phonétiques ont toutes été effectuées par des étudiants de créole de l'UAG, à l'exception de Vieux-Fort, en Guadeloupe, faite par le professeur Robert Damoiseau et de Trinité par Jo-Anne Ferreira. Une fois le nombre de 48 points définitifs retenu, j'ai récupéré l'ensemble des transcriptions et saisi moi-même l'intégralité des données sous Excel, constituant ainsi une banque de données de près de 40000 lignes.

\section{Les informateurs}

On a interrogé 92 informateurs, dont 60 hommes et 32 femmes. On est frappé par l'usage très répandu des surnoms : «A. V. dit Tarzan», «J.-L. A. dit Armand». Nous avons des renseignements biographiques assez complets sur chacun d'entre eux, sauf pour la Dominique, où les enquêteurs ont travaillé dans l'urgence et se sont parfois trouvés confrontés à plusieurs locuteurs assez bavards. Le degré d'instruction des personnes interrogées est principalement primaire, mais on trouve également quelques enseignants et fonctionnaires. Parmi les personnes nées jusque dans les années 1930 on compte plusieurs analphabètes (5 en Guadeloupe, par exemple) qui n'ont jamais fréquenté l'école. Nombre d'entre eux sont unilingues ou ont des difficultés à s'exprimer en français. Les plus jeunes ont un niveau d'instruction supérieur, certains ayant des CAP, un BTS ou des diplômes universitaires. La plupart sont agriculteurs, ouvriers agricoles, pêcheurs, maçons, charpentiers, cuisiniers, manutentionnaires... mais un certain nombre, surtout dans les îles exanglaises, a exercé des professions parfois assez prestigieuses. 


\section{Cartographie}

Le fond de cartes de base a été dessiné par Gilles Couix de l'UBO, puis revu au fur et à mesure de l'avancée du travail par Guylaine Brun-Trigaud, dialectologue romaniste du labo Bases et corpus de Nice où elle participe au projet THESOC ${ }^{11}$. Nous avons décidé de présenter les faits bruts à la Gilliéron, mais d'y adjoindre des explications étymologiques, phonétiques et grammaticales ainsi qu'une première d'interprétation des faits. Des photos ont été incluses pour les notions exotiques (aux yeux des Européens). Un premier tri nous a permis de choisir parmi les données celles qui gagnent à être cartographiées. L'exercice impose des limites dans le nombre de caractères pouvant figurer sur les cartes, qui, en outre, doivent rester lisibles. Les données trop uniformes ou fragmentaires sont intégralement conservées pour être publiées sous forme de listes commentées. Nous publierons l'atlas en deux volumes comprenant 300 cartes chacun.

Les cartes sont faites grâce à un logiciel réalisé par des étudiants de l'École Nationale des Télécommunications de Brest dirigés par Ioannis Kanellos et Ludovic Tanguy. Il a ensuite été mis en forme par François Legras en vue de l'interprétation du Nouvel Atlas Linguistique de la Basse-Bretagne (Le Dû, 2001). Enfin, Guillaume Salou, étudiant en informatique à Brest, l'a transformé pour les besoins du CD Ichtyonymie bretonne (I. Le Berre et J. Le Dû, 2010), et ensuite pour ceux de l'ALPA. Ce logiciel permet de tracer les cartes phonétiques, mais également des cartes interprétatives en couleur : l'utilisateur a la possibilité de répartir les données à sa guise, ce qui permet très rapidement de vérifier des hypothèses quant à la répartition des faits sur le terrain (Le Dû et alii, 1999).

11. Thesaurus occitan. 


\section{Présentation de quelques cartes}

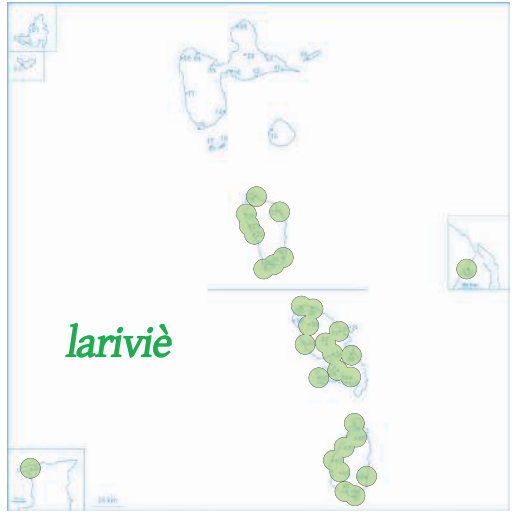

Fig. 1 : 'rivière' se dit [larivjع] - avec l'article français agglutiné - partout sauf en Guadeloupe où l'on utilise le

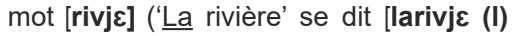
a] avec l'article postposé). Cette île semble avoir un lexique plus "moderne» que les autres îles, ce qui reste à expliquer (différence de peuplement, dates différentes d'arrivée des colons, renouvellement du peuplement?).

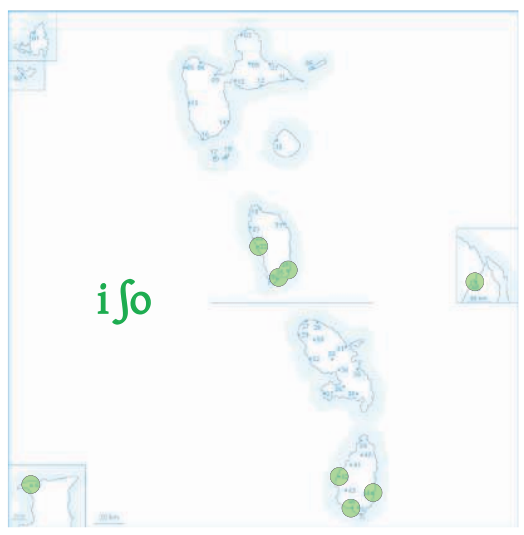

Fig. 3 : 'Il fait chaud' a pour forme simple [i Jo] 'litt. 'il chaud'. Les îles françaises ont tendance à calquer le français 'il fait chaud' en disant par exemple [i ka f $\varepsilon$ jo].

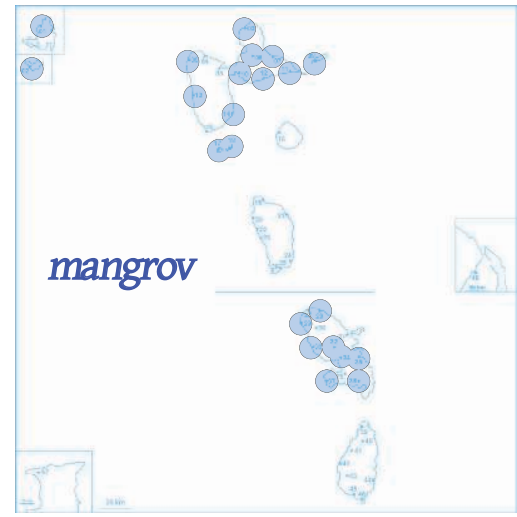

Fig. 2 : Pour 'mangrove' on a le mot français dans les îles françaises, tandis que dans les îles ex-anglaises on relève souvent des dérivés de mangue ou de palétuvier. On a souvent cette opposition entre îles françaises et îles ex-anglaises.

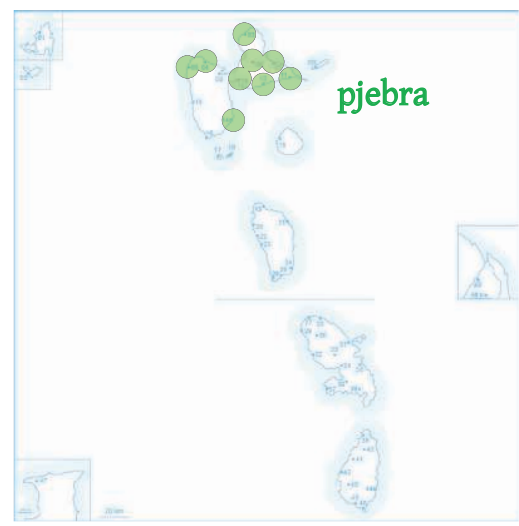

Fig. 4: 'arbre' se dit généralement [pjebwa] (litt. pied-bois). En Guadeloupe, la confusion courante [w] / [r] e entraîne des faux rétablissements: ainsi, dans certains points, [bwa] signifie 'bras' et [bra] 'bois' ! 


\section{Conclusion}

Ce travail est la première tentative d'étude géolinguistique de cette région. Il a été réalisé avec des moyens modestes, et il est évident qu'il ne constitue qu'un début. Le questionnaire, limité à des notions élémentaires, ne se préoccupe pas de relever des données ethnographiques, dont le recueil nécessiterait un investissement bien plus grand en personnel spécialisé. L'exemple de ce qui a été fait pour la Réunion montre que le champ d'étude est vaste...

L'analyse et l'interprétation des données figurant sur les cartes ne sont qu'une première approche, fort modeste. Il faudra reprendre ces données et les interpréter de diverses manières : comparaison des diverses configurations cartographiques, recours à l'histoire humaine et économique de la région pour tenter de reconstituer la genèse de la situation dialectale actuelle, etc.

Les enquêtes devraient être étendues à des zones non encore explorées de ce point de vue. Si nous disposons de l'Atlas détaillé d'Haïti (Fattier, 2000), nous n'avons rien (en-dehors de nos données sur Oiapoque) concernant le continent sud-américain : le créole de Guyane devrait être étudié de façon approfondie, mais aussi les poches créolophones des pays voisins. La Louisiane représente un terrain complexe qui doit être exploré de ce point de vue en priorité, étant donné l'état d'abandon rapide de la langue. Tout cela permettrait une comparaison sur une vaste échelle nécessitant la mise en route d'un programme plurinational ambitieux.

Notre longue fréquentation des atlas nous a appris que ceux des lecteurs qui sont eux-mêmes des locuteurs d'une variété des parlers explorés se précipitent sur le point le plus proche de leur zone et critiquent sévèrement les données qui y figurent. Il faut beaucoup de temps et de patience pour admettre que tous les mots existants n'ont pas été relevés, et que l'ouvrage comporte parfois des inexactitudes ou même des erreurs. Un atlas linguistique n'est pas un concentré de monographies locales (celles-ci doivent venir en complément) : il constitue avant tout un balayage - certes peut-être imparfait, mais minutieux - de toute une région. Nous sommes à chaque fois émerveillés, en arrivant au stade de l'interprétation, de voir se 
dessiner des zones cohérentes au sein d'un ensemble qui paraît, au premier abord, un fourre-tout dans lequel des variantes apparemment aléatoires semblent semées au hasard.

\section{Bibliographie}

CAlvet Louis-Jean \& Chaudenson Robert, Saint-Barthélemy: une énigme linguistique, Paris, CIRELFA, Agence de la Francophonie, 1998.

Chaudenson Robert, Des Iles, des Hommes, des Langues, Paris, L'Harmattan, 2000.

DEGRAFF Michel, "Linguists' most dangerous myth. The fallacy of Creole Exceptionalism", Language in Society, 34.4, 2005.

FATTIER Dominique, Contribution à l'étude de la genèse d'un créole : l'ATLAS linguistique d'Haïti, cartes et commentaires, ANRT, 1998.

Le DÛ Jean, LEGRAS François, KANELLOS Ioannis, TANGUY Ludovic, «Assistance informatique à l'interprétation des données en cartographie linguistique - informatisation anthropocentrée du Nouvel Atlas Linguistique de la Basse-Bretagne», Géolinguistique, 8, Centre de dialectologie, Université Grenoble 3, 1999, p. 181-196.

LE DÛ Jean, Nouvel Atlas Linguistique de la Basse-Bretagne, CRBC, Brest, mai 2001, 2 vol., 1999.

Le Dû Jean, Brun-Trigaud Guylaine, Atlas linguistique des Petites Antilles (vol. 1), Paris, CTHS, 2011.

LefebVRE Claire, "Substratum Semantics in the Verbal Lexicon of Haitian Creole”, Studies in Language, 23 (1), 1999, p. 61-103.

McWhORTER John H., "Identifying the creole prototype : Vindicating a typological classe", Language 74, 1998, p. 788-818.

McWhORTER John H. et PARKVALL Mikael, «Pas tout à fait du français : une étude créole», Études Créoles, vol. XXV, n 2, 2002, p. 179231.

MARCH Christian, Le discours des mères martiniquaises, diglossie et créolité : un point de vue sociolinguistique, Paris, L'Harmattan - Sémantiques, 1996.

MUFWENE Salikoko, "Genetic linguistics and genetic creolistics, short note", Journal of Pidgin and Creole Languages 18, 2003, p. 273288. 